\title{
Effect of temperature and humidity on droplet deposition of unmanned agricultural aircraft system
}

\author{
Haixia Qi ${ }^{1,2^{*}}$, Zhenxin Lin ${ }^{1,2}$, J ingkang Zhou ${ }^{1,2}$, J ianwen Li, Penchao Chen ${ }^{2,3}$, Fan Ouyang ${ }^{2,3}$ \\ (1. College of Engineering, South China Agricultural University, Guangzhou 510642, China; \\ 2. National Center for International Collaboration Research on Precision Agricultural Aviation Pesticides, Spraying Technology (NPAAC), \\ South China Agricultural University, Guangzhou 510642, China; \\ 3. College of Electronic Engineering, South China Agricultural University, Guangzhou 510642, China)
}

\begin{abstract}
Meteorological factors are one of the important factors that affect the distribution characteristics of droplet deposition and drift in unmanned agricultural aircraft system spraying operations. Among meteorological elements, there are many studies about the effects of wind speed, but there are few studies about other variables such as temperature and humidity. In order to reveal the effect of temperature and humidity on the droplet deposition parameters of unmanned agricultural aircraft system spraying operations, this study designed an experiment based on the following conditions: (1) a wind tunnel lab environment that can well control the environmental wind speed conditions; (2) UAV indoor orbit operation platform that can greatly reduce the difference in UAV flight status; (3) Guangzhou's environment with great variable temperature and humidity in December. The results show that the influence of air pressure and light on the droplet deposition is not significant; with the increase of the environmental humidity, the droplet size, coverage, and deposition amount will increase; within the temperature range of $10 \sim 29^{\circ} \mathrm{C}$, the effect of temperature conditions on droplets deposition is not significant. The research results can provide guidance for the practical production and application of agricultural aviation to reduce droplet loss and improve pesticide utilization.
\end{abstract}

Keywords: temperature, humidity, plant protection UAV, spray, droplet deposition

DOI: $10.33440 /$ j.ijpaa.20200303.100

Citation: Qi H X, Lin Z X, Zhou J K, Li J W, Chen P C, Ouyang F. Effect of temperature and humidity on droplet deposition of unmanned agricultural aircraft system. Int J Precis Agric Aviat, 2020; 3(3): 41-49.

\section{Introduction}

The use of unmanned agricultural aircraft systems for pesticide spraying is an important means to improve the utilization rate of pesticides and fertilizers. Compared with manual spraying, UAV application has route planning function, and can even spray pesticides and chemical fertilizers according to crop conditions, which can significantly reduce repeated spraying and further reduce the use of pesticides ${ }^{[1,2]}$. The droplet drift of UAV operations is mainly affected by four factors: meteorological parameters, spray application technology, other surrounding environment characteristics, and the physicochemical nature of the spray liquid $^{[3]}$. Among them, the meteorological parameters mainly include temperature, humidity and wind speed. High temperatures can accelerate the evaporation of droplets, reduce the

Received date: 2020-08-01 Accepted date: 2020-09-17

Biographies: Zhenxin Lin, Postgraduate, research interests: deep learning, agriculture informatization, Email: lin_zhenxin@qq.com; Jingkang Zhou, Postgraduate, research interests: The application of variable spray technology in UAV, Email: 18819268227@163.com; Jianwen Li, Postgraduate, research interests: Agricultural Aviation Application Technology, Email: 670510490@qq.com; Pengchao Chen, PhD Student, research interests: Spray Application of UAV, Precision Agriculture, Email: pengchao@stu.scau.edu.cn; Fan Ouyang, Lecturer, research interests: research and application of open-source agricultural UAV (Unmanned Aerial Vehicle) platform combined with robotic technology, Email: ouyangfan@scau.edu.cn.

*Corresponding author: Qi Haixia, $\mathrm{PhD}$, Associate Professor, research interests: intelligent agriculture and system, precision agricultural aviation spray and efficacy evaluation, South China Agricultural University, Wushan Road, Tianhe District, Guangzhou City, Guangdong, 510642, China. Email: qihaixia_scau@126.com. size of droplets, and even completely evaporate droplets with smaller particle diameters. Humidity refers to the amount of water vapor in the air. High humidity can cause the bigger size of droplets in the air due to moisture absorption. Low humidity may cause the droplets in the air to shrink due to the diffusion of moisture. The wind speed can change the drift path of the droplets, which will also change the deposition parameters of the droplets.

Regarding the influence of wind speed on the drift and deposition of droplets, there have been many previous studies. For example, Zhang et al. ${ }^{[4]}$ studied the spray characteristics of unmanned agricultural aircraft system centrifugal nozzles under different wind speed conditions and found that the drift amount increased as the increase of wind speed and decreased of $D_{V 50}$. Tang et al. ${ }^{[5]}$ research under the conditions of different spray pressure and wind speed, the drift of the air intake nozzles and indicated that the value $D_{\mathrm{V} 10}$ and $D_{\mathrm{V} 50}$ of the droplet decrease approximately linearly with the increase of wind speed, while $\mathrm{D}_{\mathrm{V} 90}$ is affected by the square of the wind speed. $\mathrm{Lv}$ et al. ${ }^{[6]}$ used infrared thermal imaging technology to explore the flight speed and droplet deposition density, droplet deposition coverage and the relationship between the average value of arithmetic droplet size and the result shows that the droplet diameter variation coefficient $\mathrm{CV}$ increases with the increase of the UAV flight speed, resulting in the bad uniformity of droplet distribution. In terms of constructing the model. Wang et al. ${ }^{[7]}$ studied the droplet deposition model based on UAV spraying operation and it suggests that Wind speed, flying height and droplet diameter are the most important factors while the spray angle of the nozzle has no significant effect on the deposition distribution. $\mathrm{Ru}$ et $\mathrm{al}^{[8]}$ 
studied the droplet drift model under wind tunnel conditions and the model suggests that as the increase of air velocity, the drift distance of the droplets increases significantly, and the droplets smaller than $200 \mu \mathrm{m}$ are more likely to drift under the effect of crosswind. Teske et al. ${ }^{[9]}$ studied the evaporation rate of the isolated water droplets and indicated that as the Reynolds number (the relative velocity between the droplet and the surrounding air) decreases toward zero, the evaporation rate of the droplet drops to half of its value. Most of the related research is based on wind tunnel conditions, which can well control the fluctuation of wind speed conditions.

Many research works have proven that wind speed condition has a huge impact on the droplet deposit parameter. But there are little studies of the impact of temperature and humidity on them. In terms of temperature and humidity, it has been known that temperature and humidity are closely related to pesticide efficacy and pests and diseases ${ }^{[10,11]}$. And the study will benefit agricultural application efficiency.

Temperature and humidity have a great relationship with the size and state of spray droplets. In terms of temperature, Alizadeh et al. ${ }^{[12]}$ studied the impact dynamics of temperature-dependent droplets on flat and textured surfaces, and found that droplet retraction exhibits a very strong temperature dependence, especially in the hydrophilic surface. Ryumin et al. ${ }^{[13]}$ studied the effect of ambient temperature on the formation process and characteristics of droplet clusters, and the result shows that a moderately localized heated water surface can also produce sufficiently large self-assembled levitating clusters of water droplets. Mousavi et al. ${ }^{[14]}$ evaluated the effects of viscosity, density and surface tension on droplet dynamics under the temperature dependence of each element, and they found that Newtonian and non-Newtonian physicochemical liquid properties are greatly affected by temperature. Jin et al. ${ }^{[15]}$ analyzed the images of tiny tracer particles in water droplets under different surface temperature conditions, to clearly and quantitatively reveal the time evolution of the contact line, the contact angle, and the volume change of water droplets during the evaporation process. Zuo et al. ${ }^{[16]}$ numerically studied the evaporation of a sessile water droplet under the coupling of static electricity and high temperature, indicating that due to the interaction between the electric field force and the force caused by the temperature gradient, the flow rate will be reduced and the lower flow rate limits the evaporation rate of droplets in the high-temperature field.

In terms of humidity, Portuguez et al. ${ }^{[17]}$ used the hanging drop method and combined image analysis to show the change of the surface tension of deionized water with relative humidity under the temperature conditions of $293 \sim 343 \mathrm{~K}$, indicating that humidity affects its surface tension. Kubochkin et al. ${ }^{[18]}$ found that periodic changes in relative humidity can cause hysteresis differences in different droplets. Jadidi et al. ${ }^{[19]}$ found that the ratio of surface temperature to dew point temperature (depending on air temperature and relative humidity) has a great influence on droplet dispersion, recoil force and contact angle. Bugarin et al. ${ }^{[20]}$ modeled the interaction between gas turbine compressor blades and air droplets to quantify the relationship between relative humidity and total heat transfer coefficient and blade surface temperature. BouZid et al. ${ }^{[21]}$ studied the influence of relative humidity (in the range of $20 \%$ to $80 \%$ ) and viscosity on the spreading kinetics of water-glycerol mixtures and established a newer Tanner's law.

The above research indicates that temperature and humidity are likely to have a great impact on the droplet deposition of plant protection drone spraying operations. However, few people have studied the impact of the environmental temperature and humidity on the droplet deposition of UAV operations. There are two reasons for this: The first is that the surrounding wind speed conditions must be well controlled. In this regard, it is necessary to find a continuous and windless large-scale environment for the UAV to operate stably. The second is the unmanned flight status, such as horizontal speed, altitude, etc. must be well controlled, a little carelessness will cause some flight parameters to change and cause errors. The use of wind tunnel laboratory conditions can have better control over environmental wind conditions, but it cannot control the characteristics of environmental temperature and humidity. And the use of indoor orbital platforms which can carry UAV to run can greatly reduce parameter errors in the flying process. We have designed the UAV spray experiment to study the influence of environmental temperature and humidity on the droplet deposition of UAV spraying operations based on the above two conditions.

\section{Materials and Methods}

This experiment was conducted from December 11, 2018, to January 2, 2019, at Qilin North Wind Tunnel Laboratory of South China Agricultural University. During this time period, due to the influence of the cold wave, the meteorological conditions have undergone relatively drastic changes, and the temperature and humidity changes are relatively large. The wind tunnel laboratory covers a large area, which can shield the influence of natural wind and some other natural meteorological conditions on the experiment.

\subsection{Experiment platform}

Wind Tunnel Lab has an indoor orbital operating platform that can carry UAV. The platform is $2.86 \mathrm{~m}$ high and $10 \mathrm{~m}$ long. The UAV can move linearly on the platform, and the moving speed can be controlled between $0 \sim 0.9 \mathrm{~m} / \mathrm{s}$. As shown in Figure 1, the test aircraft is a P20 imitation UAV, the nozzle type is a pressure nozzle, and the nozzle model is Lechler110-01.

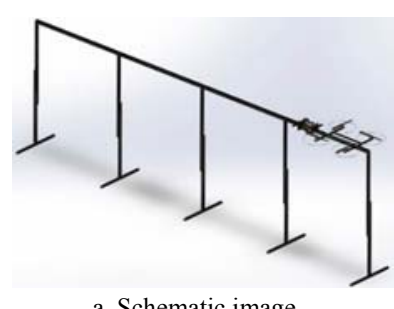

a. Schematic image

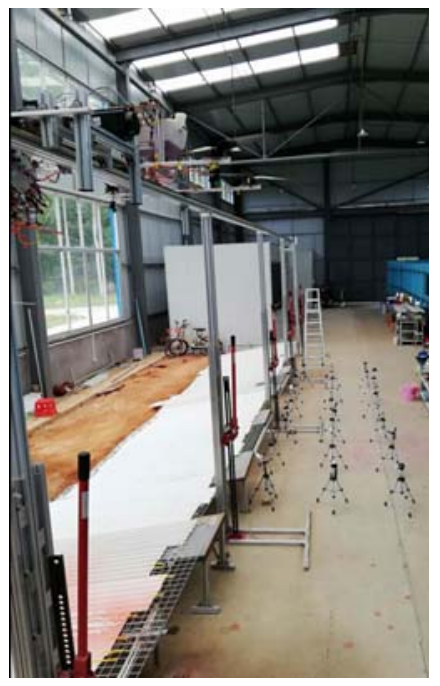

b. Physical images
Figure 1 Indoor orbital operating platform

\subsection{Environment meteorology information measurement}

In the experiment, the values of ambient temperature, humidity, illuminance, and air pressure during the experiment have been recorded. It is of course required to record temperature and humidity. And because of the different experiment time, the illuminance and air pressure are also different and also need to be 
recorded. For example, the air pressure is generally lower in the afternoon, and the light intensity is stronger in that time. Recording the ambient light and air pressure is to exclude the influence of other environmental meteorological factors on the experiment.

The electronic temperature and humidity meter produced by GEMlead (as shown in Figure 2a) was used as the measuring instrument for ambient temperature and humidity. The range is $20 \sim 90 \% \mathrm{RH}$, and the error is $\pm 5 \% \mathrm{RH}$, which is in line with the Chinese temperature and humidity meter standard (JJG 047-2017).

The measuring instrument selected for the illuminance is the
PM6612L digital LED illuminance meter (as shown in Figure 2b), which was produced by Shenzhen Huayi Peakmeter Technology Co., Ltd. Its measuring illuminance range is $0 \sim 200,000$ Lux, the accuracy is $3 \%$, and the resolution is 0.01 Lux. The operating temperature range is $-10 \sim 50^{\circ} \mathrm{C}$, and the humidity is $<80 \% \mathrm{RH}$.

The measuring instrument for air pressure is YBS-C precision digital absolute pressure meter (as shown in Figure 2c). The product is made in Jiansu Mingyu Automatic Control Equipment Co. Ltd.. Its range is $0-150 \mathrm{kPa}$, and its accuracy is $\pm 1 \mathrm{~Pa}$. The operating environment temperature range is $5 \sim 50^{\circ} \mathrm{C}$, humidity $\leq$ $80 \%$ RH.

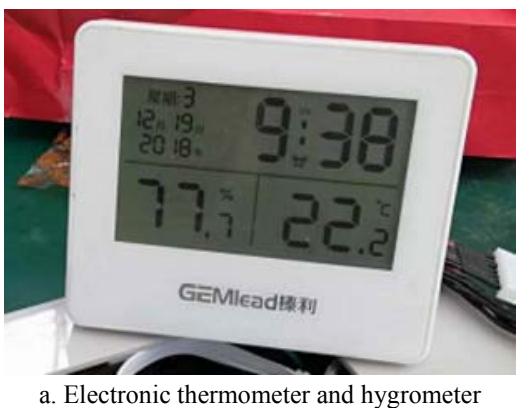

a. Electronic thermometer and hygrometer

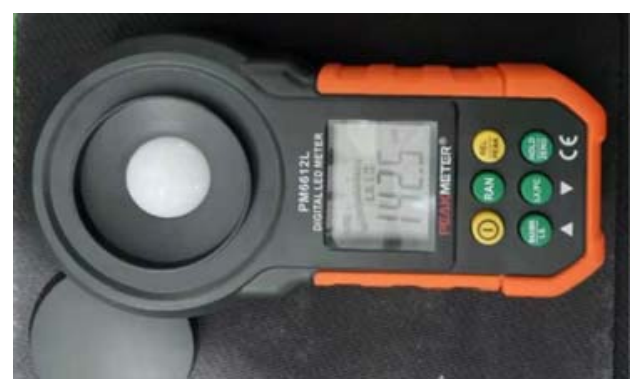

b. Illuminance meter

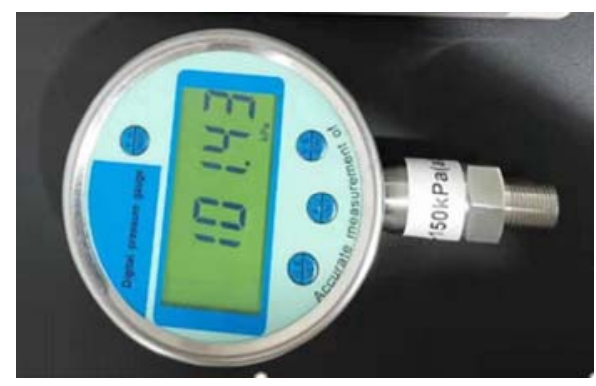

c. Absolute pressure gauge

Figure 2 Meteorological information measuring instrument

\subsection{Design of experiment}

This experiment detects whether temperature and humidity have an effect on the droplet size, droplet density, deposition amount, and coverage of UAV spraying operations. In order to control variables, during the experiment, the UAV throttle was kept at no-load throttle for the amount of liquid in each test is small (2 $\mathrm{L} / 10 \mathrm{~L}$ ) and then turn on two of the nozzles (one on each side of the track) and the distance between the two nozzles is $1 \mathrm{~m}$. The flow rate of each nozzle is $234 \mathrm{~mL} / \mathrm{min}$. The running speed of UAV is $0.9 \mathrm{~m} / \mathrm{s}$ under the control of the backside small car.

As shown in Figure 3, the track platform is used as the running route. And the route contains 3 parts: the part where the car is accelerating ( $1 \mathrm{~m}$, part 1$)$, the part where the car is running at a constant speed (6 $\mathrm{m}$, part 2), and the part where the car is decelerating ( $1 \mathrm{~m}$, part 3$)$. During the experiment, the car carries the UAV at speed from 0 to $0.9 \mathrm{~m} / \mathrm{s}$ in part 1 and runs at a constant speed at part 2 and decelerate until it stops running in part 3 . The coated art papers $(30 \mathrm{~mm} \times 80 \mathrm{~mm})$ are evenly distributed in the middle $5 \mathrm{~m}$ of part 2. A total of three lines of coated art papers are placed, with the first row place closest to the track and the second and third rows rank the second and third closest respectively. In the track direction, a piece of coated art paper is arranged every one meter, and in the vertical track direction, every $0.7 \mathrm{~m}$ place a piece of coated art paper, a total of 18 pieces of coated art paper was used in each test and each coated art paper is $0.5 \mathrm{~m}$ from the ground.

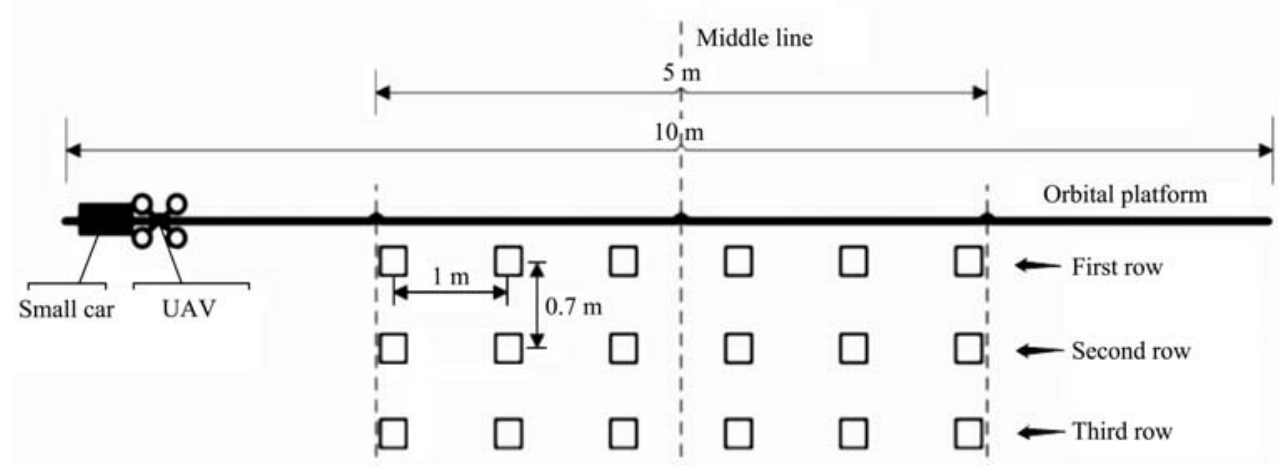

Figure 3 Schematic diagram of sample layout

The coloring agent selected in the experiment is the lure red solution, the configuration ratio is $5 \mathrm{~g} / \mathrm{L}$, and the configured solution is placed in the medicine box of the drone. Before the experiment, the temperature, humidity, illuminance, and air pressure of the surrounding environment would be recorded firstly and then the start button of the electric car would be pressed. According to the setting of the car startup program, there will be a startup time of $15 \mathrm{~s}$. During this time, first use the remote control to start the UAV, and control the throttle of the UAV within $46 \% \sim 47 \%$, and then start the spray nozzle to spray the solution. By doing this, the UAV would pass through the droplet collection area uniformly and spray the paper evenly.

Each experiment was repeated three times. Before collecting and storing the coated art paper, it had to wait for all the droplets floating in the air to settle on the coated art paper and dry. After the experiment is completed, first use the HP scanner to scan the coated art paper, and then use the image processing software DepositScan to analyze the droplets on the coated art paper, getting the value of droplet size, coverage, deposit amount and droplet density

\subsection{Experimental method}

The test was conducted in December in Guangzhou, which belongs to the Lingnan area of China. The area is often hit by strong cold air in December. The temperature will suddenly drop when the cold air transits, and it will be accompanied by weather phenomena such as rain. The duration of cold air transit is 
generally 5-10 days and after the cold air transits, the temperature will gradually pick up. Therefore, it is easy to collect the weather information with a large temperature difference and large humidity difference in December, which is very suitable for experimental research on the influence of temperature and humidity.

The temperature and humidity series tested in this experiment are shown in Table 1. Each experiment was repeated 3 times, getting a total of 54 sets of data. Using SPSS19.0 software for variance analysis and Origin Pro8.0 software for graphing respectively.

Table 1 Experimental temperature and humidity series

\begin{tabular}{ccc}
\hline Experiment number & Temperature $/{ }^{\circ} \mathrm{C}$ & Humidity $/ \% \mathrm{RH}$ \\
\hline 1 & 10 & 60 \\
2 & 10 & 60 \\
3 & 19 & 50 \\
4 & 19 & 50 \\
5 & 24 & 33 \\
6 & 24 & 46 \\
7 & 24 & 55 \\
8 & 24 & 68 \\
9 & 24 & 77 \\
10 & 13 & 54 \\
11 & 19 & 54 \\
12 & 29 & 54 \\
13 & 11 & 68 \\
14 & 28 & 68 \\
15 & 15 & 45 \\
16 & 24 & 45 \\
\hline
\end{tabular}

Among them, the temperature and humidity are the same in the serial numbers of 1 and 2, 3 and 4 . But because the measurement time points are different, their corresponding pressure, light intensity and other conditions are not the same. The results of the experiments corresponding to these serial numbers can be used to exclude the influence of other meteorological factors or something else on droplet deposition.

Experiments with serial numbers 5 9 can verify the effect of humidity conditions on droplet deposition for their same environment temperature and different humidity; experiments with serial numbers $10 \sim 16$ can verify the effect of temperature on droplet deposition for their same humidity value and different temperature value.

\subsection{Experiment processing}

In order to study the influence of temperature and humidity on the droplet deposition of UAV spraying, the evaluation indicators used in this experiment are droplet size, coverage, droplet density, and deposition amount. Among them, the droplet size is represented by $\mathrm{D}_{\mathrm{V} 10}, \mathrm{D}_{\mathrm{V} 50}$, and $\mathrm{D}_{\mathrm{V} 90}$. It means that if the droplets are sorted according to the diameter size from small to large, $\mathrm{D}_{\mathrm{V} 10}, \mathrm{D}_{\mathrm{V} 50}$, and $\mathrm{D}_{\mathrm{V} 90}$ are the droplet size values which respectively located at $10 \%, 50 \%$, and $90 \%$ of the total sequence and its unit is " $\mu \mathrm{m}$ "; the coverage is the percentage of the coverage area of the liquid solution in per unit paper area; the droplet density is the number of droplets in a square centimeter; the deposition amount is the deposition amount of the liquid solution in per unit area, the unit is " $\mu \mathrm{L} / \mathrm{cm}^{2} "$. During the process of data processing, each experiment has three sets of replicates, and each replicate has six sets of data. We compare all 18 sets of data in each experiment.

\section{Test results and analysis}

3.1 Measurement results under the same temperature and humidity conditions
This section compares the results of two pairs of experiments: experiments with numbers of 1 and 2 , and experiments with numbers of 3 and 4 . The ambient temperature and humidity of experiments 1 and 2 are the same, which are $10^{\circ} \mathrm{C}$ and $60 \% \mathrm{RH}$, respectively. But the average illuminance of experiment 1 was $20 \mathrm{Lux}$ and the air pressure was $108 \mathrm{kPa}$, while experiment 2 was $150 \mathrm{Lux}$ and $103 \mathrm{kPa}$. The surrounding temperature and humidity of experiments 3 and 4 are also the same, respectively $19^{\circ} \mathrm{C}$ and $50 \% \mathrm{RH}$, while experiment 3 is $10 \mathrm{Lux}$ and $108 \mathrm{kPa}$, and experiment 4 is 260 Lux and $105 \mathrm{kPa}$. The experimental results can verify whether the illuminance and air pressure or other environmental meteorological factors will affect the droplet deposition parameters.

The experimental results are shown in Figures 4, 5. The data of the experiment is analyzed using Student's t test in the SPSS software.

The experimental results are shown in Figure 4 and Figure 5. From Figure 4 and Figure 5, it can be seen that the differences in the experimental data of each group are basically small. It can be further seen from the figures: In the comparative experiments at environment weather of $10^{\circ} \mathrm{C}$ and $60 \% \mathrm{RH}$ and in the comparative experiments at environment weather of $19^{\circ} \mathrm{C}$ and $50 \% \mathrm{RH}$, all data are at $95 \%$ significance level and $99 \%$ significance level showed no difference.

It can be seen from the data results that other meteorological conditions such as light intensity and air pressure have no significant effect on the droplet size, coverage, droplet density, and deposition amount of UAV spraying operations. This result can lay the foundation for the following research on the influence of temperature and humidity on spray droplet parameters.

\subsection{Experiment results under the same temperature and different humidity conditions}

This section compares the data of 5 experiment results: experiments with numbers of 5 to 9 . Each experiment contains three replicates, and each replicate contains six sets of data, which are the same as before. The experimental results are shown in Figures $6,7,8$. The experimental data are humidity of $33 \% \mathrm{RH}$, $46 \% \mathrm{RH}, 55 \% \mathrm{RH}, 68 \% \mathrm{RH}$ and $77 \% \mathrm{RH}$, respectively, and analyzed by LSD method.

It can be clearly seen from Figures 6that as the humidity increases, the droplet size, coverage, and deposition amount basically increase. In the first row, both at the $99 \%$ confidence level and $95 \%$ confidence level, the droplet sizes $\left(\mathrm{D}_{\mathrm{V} 10}, \mathrm{D}_{\mathrm{V} 90}, \mathrm{D}_{\mathrm{V} 50}\right)$ of $77 \% \mathrm{RH}$ and $68 \% \mathrm{RH}$ are significantly larger than the droplet size under the environmental conditions of $33 \% \mathrm{RH}, 46 \% \mathrm{RH}$ and $55 \%$ RH.

Moreover, at two confidence levels, the coverage and deposition of $77 \% \mathrm{RH}$ and $68 \% \mathrm{RH}$ are also significantly bigger than those under the experimental conditions of $33 \% \mathrm{RH}, 46 \% \mathrm{RH}$ and $55 \% \mathrm{RH}$. However, Figure 6 also shows that at the $99 \%$ significance level, the droplet density of $77 \% \mathrm{RH}$ will be smaller than that of the other four levels and at the $95 \%$ significance level, the droplet density of $77 \% \mathrm{RH}$ and $66 \% \mathrm{RH}$ is less than the other three levels.

Figure 7 shows the deposition data of the second row of droplets. The data in the second row basically have no difference except a significant difference in the $\mathrm{D}_{\mathrm{V} 90}$ column at the confidence level of $95 \%$, which shows an increasing trend. The DV90 value of $77 \% \mathrm{RH}$ and $66 \% \mathrm{RH}$ is significantly greater than those of $33 \% \mathrm{RH}$ and $46 \% \mathrm{RH}$. 


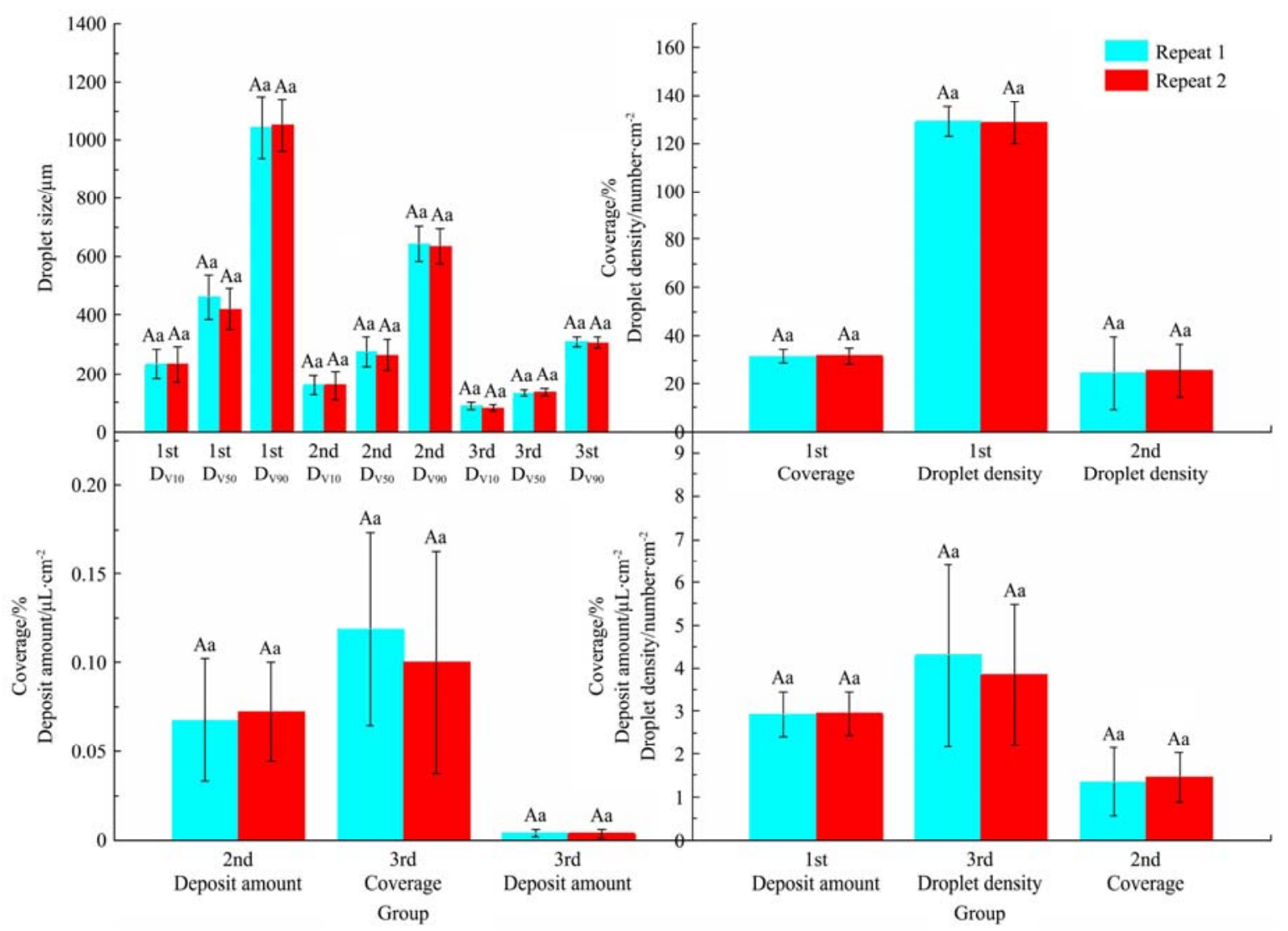

Note: The difference at the $99 \%$ significance level are indicated by different capital letters, and the differences at the $95 \%$ significance level are indicated by lower case letters. If one of the letters is different, there is a difference in the detection level of the response. It should be noted that the difference here refers to the difference between two sets of data of the same category, that is, two sets of data next to each other without space in the figure. The same of Figures 5, 9 , 10 and 11. Moreover, these figures put together data of the same order of magnitude but in different units, so it is a bit complicated: " 1 st" represents the data of the first row, " 2 nd" represents the data of the second row, and "3rd" represents the data of the third row. There are related parameters below the number of rows and its unit can be found in the left legend. What the number of rows represents can be found in Figure 3.

Figure 4 Comparison of experimental data of different groups under humidity of $60 \% \mathrm{RH}$ and temperature of $10^{\circ} \mathrm{C}$

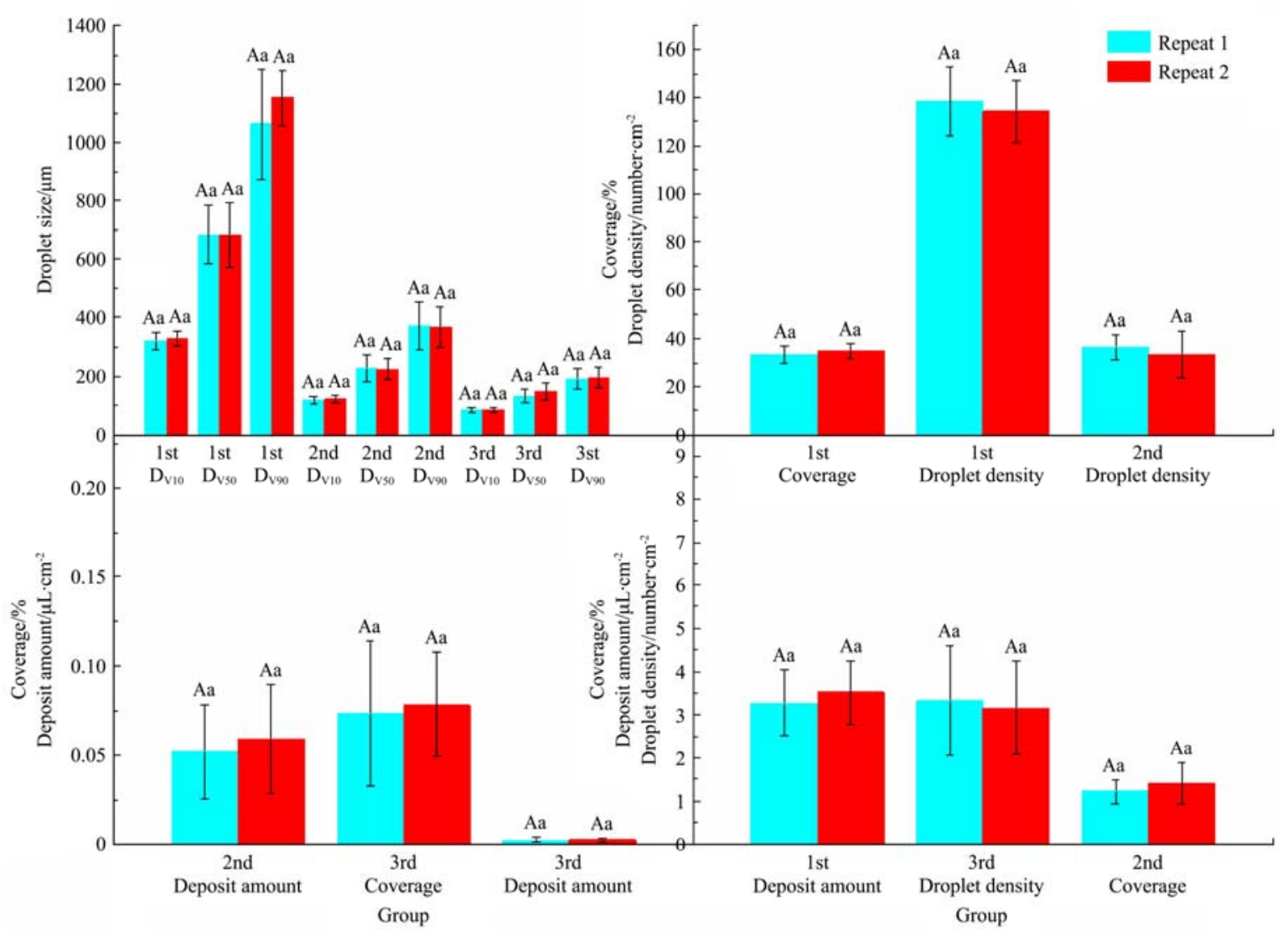

Figure 5 Comparison of experimental data of different groups under humidity of $50 \% \mathrm{RH}$ and temperature of $19^{\circ} \mathrm{C}$ 

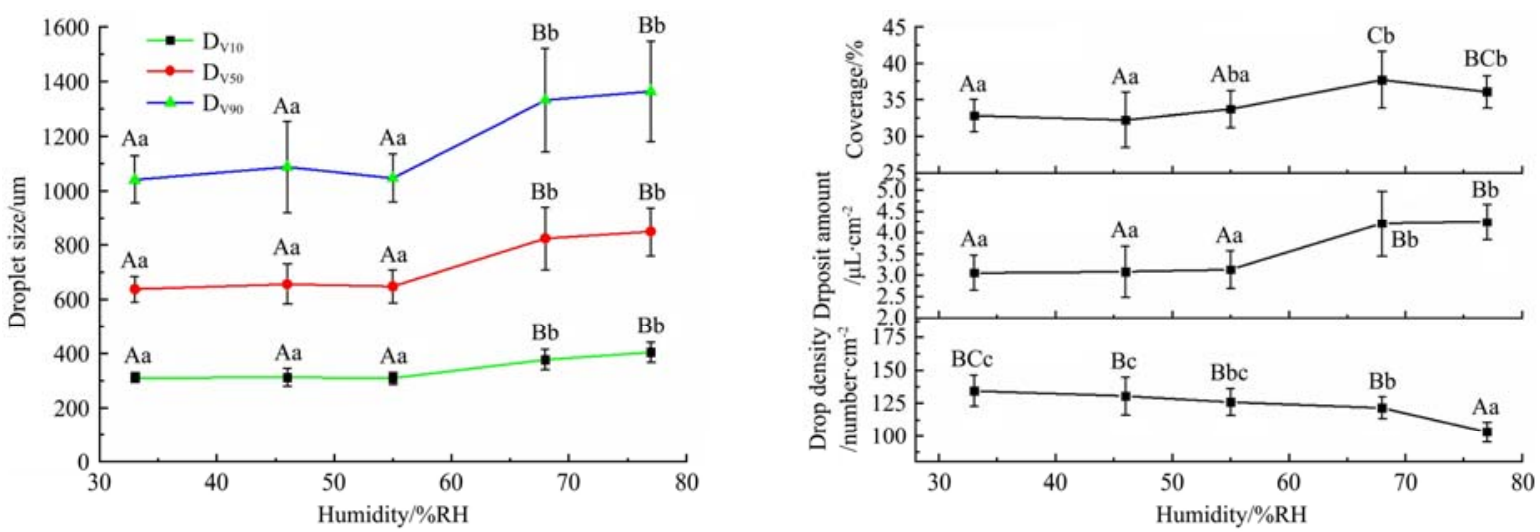

Note: The difference at the $99 \%$ significance level is indicated by different capital letters, and the difference at the $95 \%$ significance level is indicated by lower case letters. The same of Figure 7 and 8.

Figure 6 Comparison of data in the first row of experiments with the same temperature and different humidity
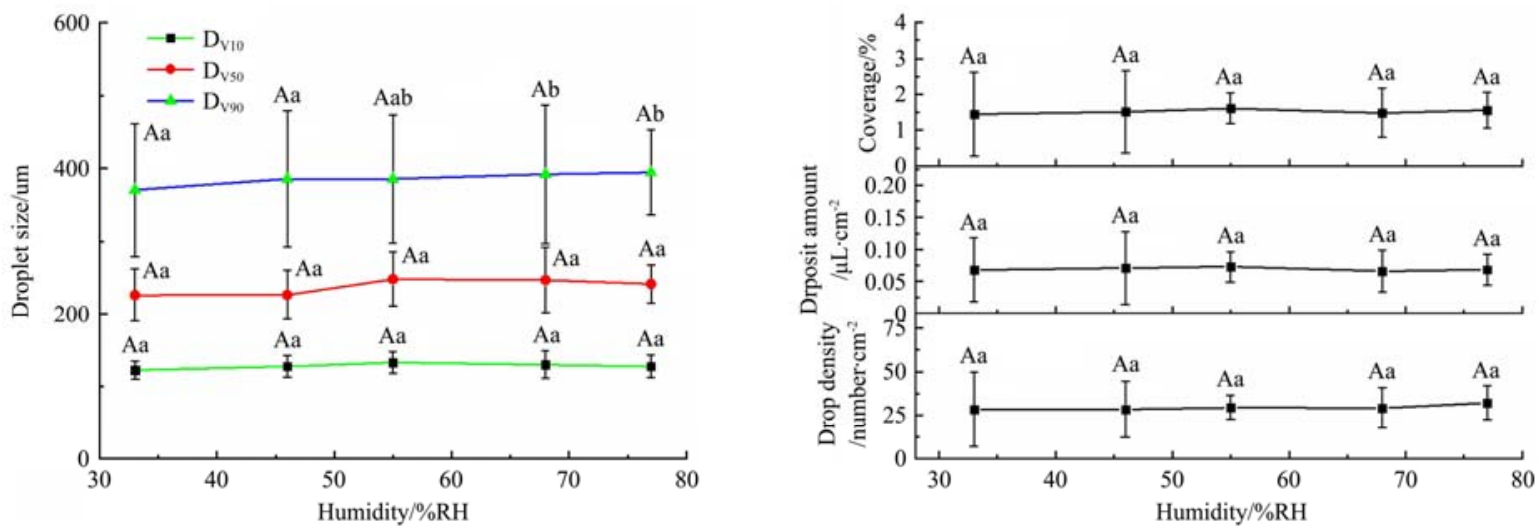

Figure 7 Comparison of data in the second row of experiments with the same temperature and different humidity
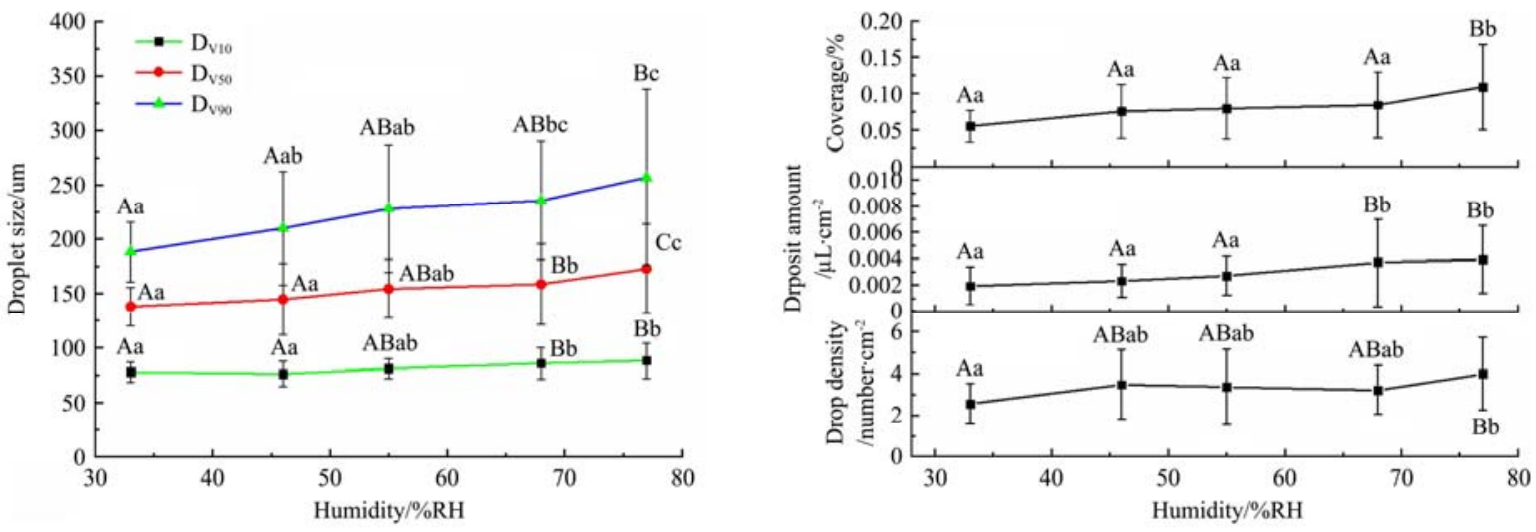

Figure 8 Comparison of data in the third row of experiments with the same temperature and different humidity

Figure 8 corresponds to the third row of droplet deposition data. As can be seen from it, the experiment data of droplet size and coverage under the $77 \% \mathrm{RH}$ experimental conditions are basically bigger than those under the other four humidity conditions at the $99 \%$ confidence level. And at this level, it also bigger than the deposit amount data of $33 \% \mathrm{RH}, 46 \% \mathrm{RH}$, and $55 \% \mathrm{RH}$, and bigger than the drop density data of $33 \% \mathrm{RH}$. All in all, At the confidence level of $95 \%$, it can also be seen that all experimental parameter data have a growing trend with the increase in environment humidity.

It can be seen from the above analysis that at a temperature of $24^{\circ} \mathrm{C}$ :

(1) The first and third rows of data will increase with the increase of humidity in general, including droplet size, coverage and deposition value of UAV spraying operation. However, the experimental results of droplet density in the first row are the opposite. Presumably, the droplet diameter of the first row is relatively large, the droplets will more likely to adhere due to the interaction, resulting in a decrease in the density of the droplets.

(2) In the data of the second row, with the increase of humidity, the $\mathrm{D}_{\mathrm{V} 90}$ of UAV spraying operation will increase significantly. But there is no obvious difference between other data. Compared with the first row and the third row, the increasing trend of each parameter is not obvious. It is speculated that it may be caused by a complicated UAV rotor wind field.

(3) The analysis of all three rows of data shows that there is an increasing trend of the droplet parameters as the humidity increases to varying degrees. It shows that with the increase of environmental humidity, the droplet size, coverage and deposition value of UAV spraying operation will increase.

\subsection{Experiment results under the same humidity and} different temperature conditions

3.3.1 Data analysis under $54 \% \mathrm{RH}$ humidity

This section shows the results of experiments of number 
10-12, which are under the conditions of humidity $54 \% \mathrm{RH}$ and temperature $13^{\circ} \mathrm{C}, 19^{\circ} \mathrm{C}, 29^{\circ} \mathrm{C}$. The result is shown in Figure 9 . The data in Figure 9 were analyzed by ANOVA with SPSS software.

As can be seen from Figure 9, under the conditions of humidity of $54 \% \mathrm{RH}$ and temperature range of $13^{\circ} \mathrm{C}$ to $29^{\circ} \mathrm{C}$, all related deposition data did not show significant differences.

3.3.2 Experiment data analysis under $68 \% \mathrm{RH}$ humidity

This section shows the result of experiments of 13-14. The data analysis under the conditions of humidity of $68 \% \mathrm{RH}$ and temperature of $11^{\circ} \mathrm{C}$ and $28^{\circ} \mathrm{C}$ is shown Figure 10 . The data in Figure 10 is analyzed by the student's t-test in SPSS.

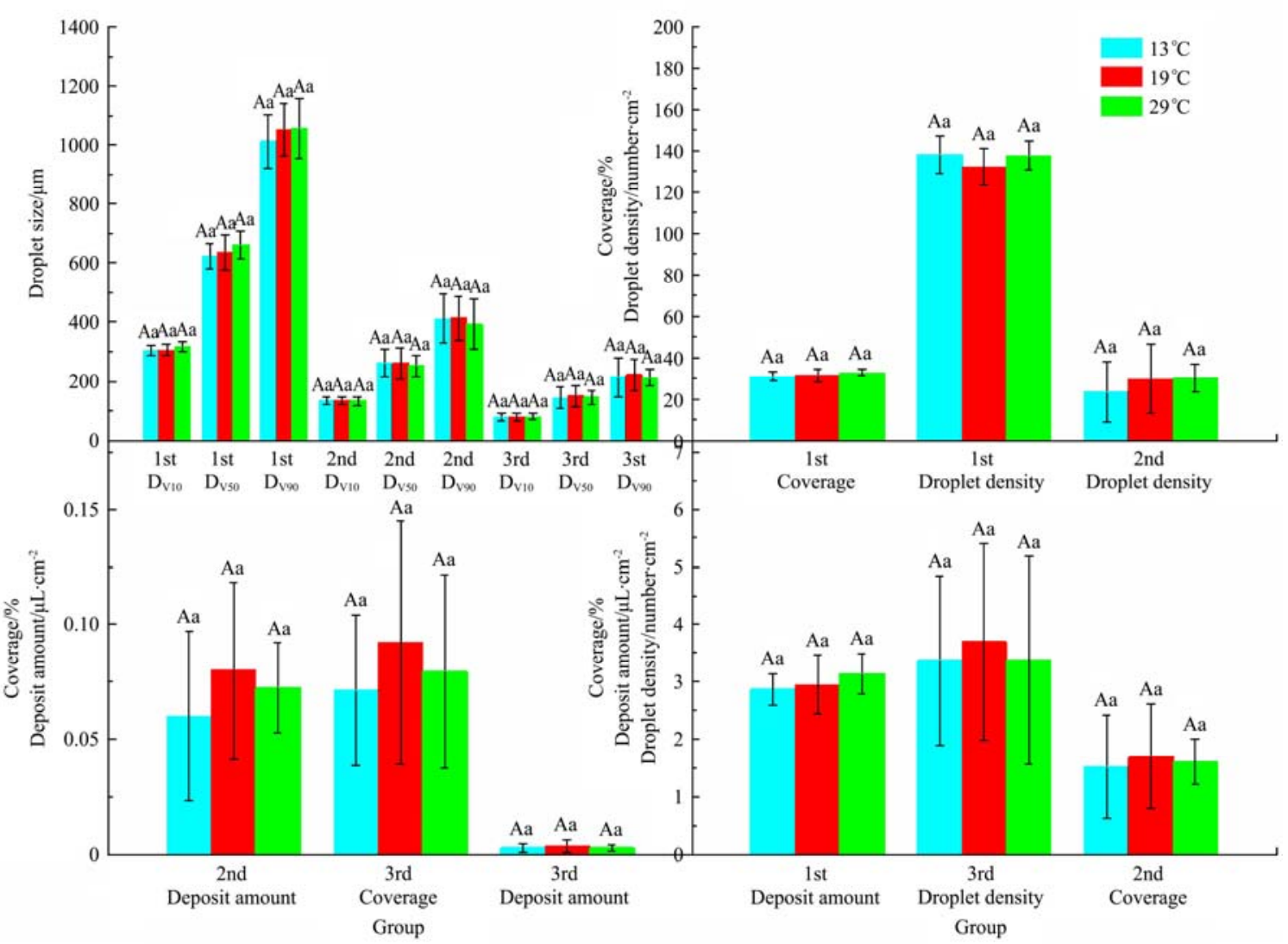

Figure 9 Comparison of experimental data under 54\%RH

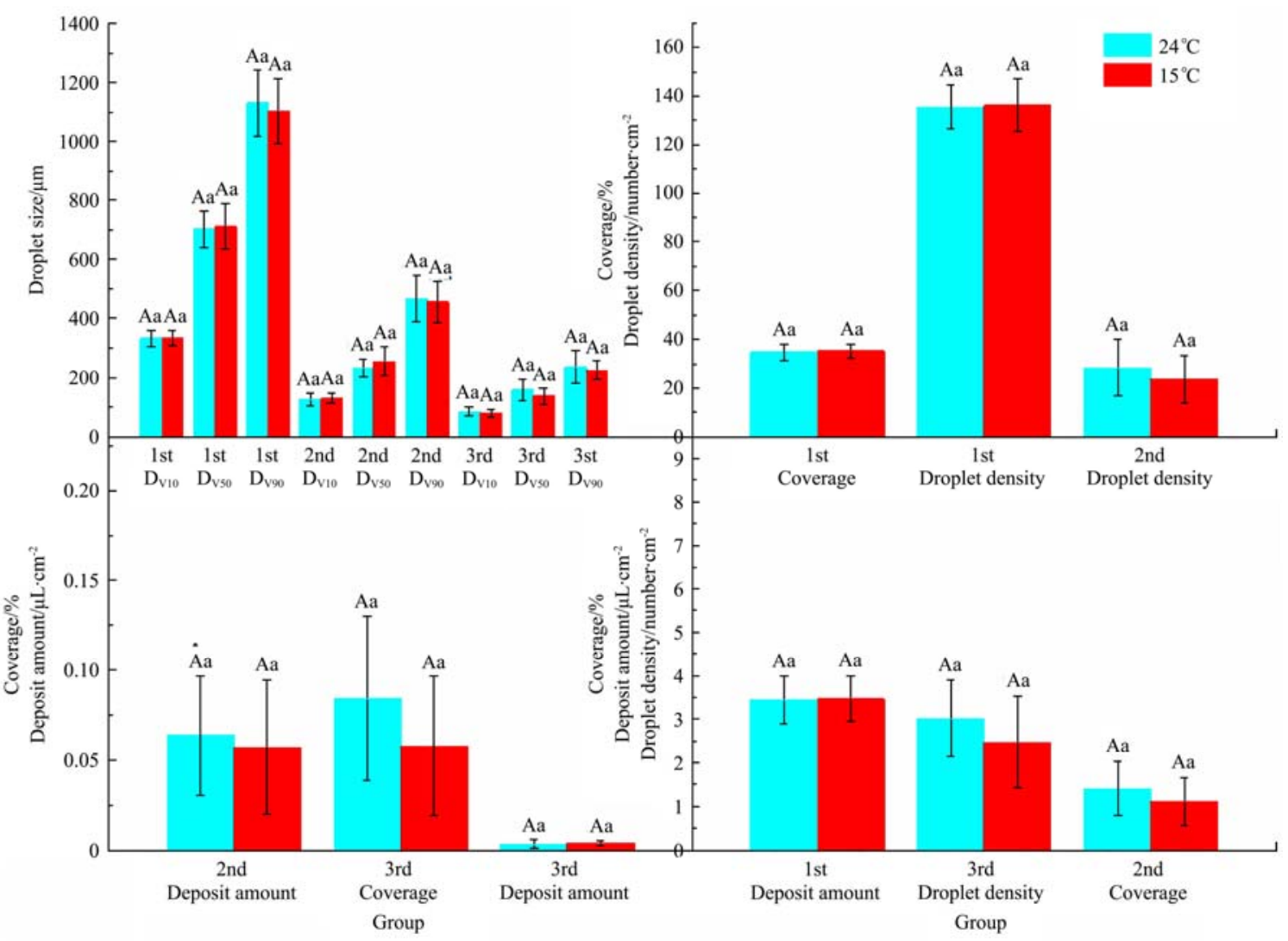

Figure 10 Comparison of experimental data under $68 \% \mathrm{RH}$ 
Under the conditions that the humidity are $68 \% \mathrm{RH}$ and the temperature are $11^{\circ} \mathrm{C}$ and $28^{\circ} \mathrm{C}$ respectively, there is no obvious difference in the comparison of the various parameter values in each row. It shows that when the humidity is $68 \%$, the temperature conditions of $11{ }^{\circ} \mathrm{C}$ and $28^{\circ} \mathrm{C}$ have no significant effect on the deposition of the UAV spraying.

3.3.3 Experiment data analysis under $46 \% \mathrm{RH}$ humidity

The data analyses under the conditions of humidity of $46 \% \mathrm{RH}$ and temperature of $15^{\circ} \mathrm{C}$ and $24^{\circ} \mathrm{C}$ are shown in Figure 11.

As can be seen from Figure 11, when the humidity are $46 \% \mathrm{RH}$ and the temperature are $15^{\circ} \mathrm{C}$ and $24^{\circ} \mathrm{C}$ respectively, no different upper-case or lower-case letters can be seen. Indicating that the difference between each condition parameter values is small. It can be considered that if the environment humidity is $46 \% \mathrm{RH}$, the two temperature of 15 and $24^{\circ} \mathrm{C}$ showed no significant effect on the deposition of droplets sprayed by the UAV.

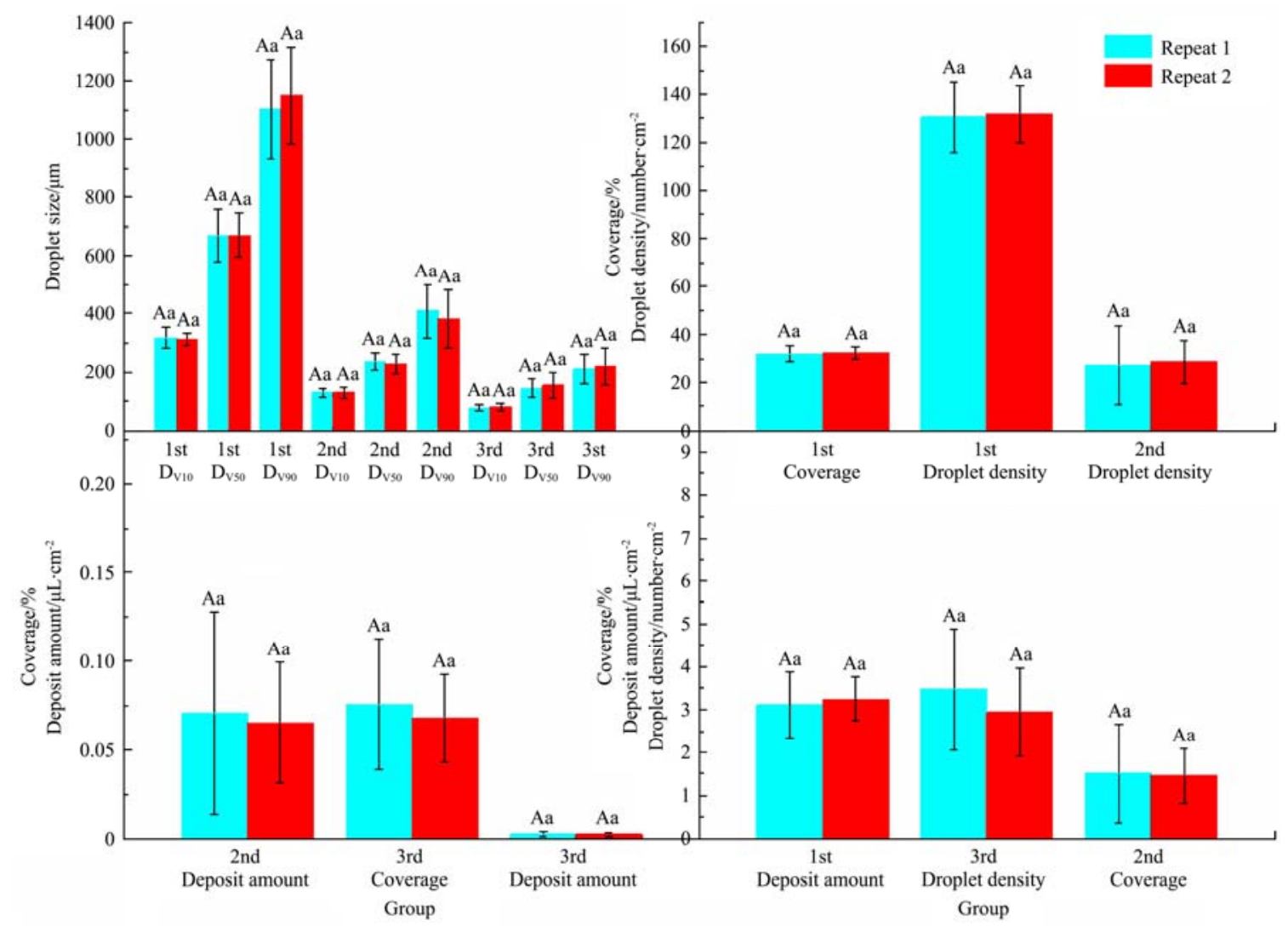

Figure 11 Comparison of experimental data under $45 \% \mathrm{RH}$

In summary, when the temperature range is $11 \sim 29^{\circ} \mathrm{C}$, the temperature has no significant effect on the droplet size, coverage, droplet density and deposition amount.

\section{Discussion}

In this paper, under different temperature and humidity conditions, the UAV test track operation platform in the wind tunnel lab was used to test droplet size, droplet deposition amount, droplet deposition density, and droplet coverage of the unmanned agricultural aircraft system spraying droplets. Exploring the effect of temperature and humidity on the deposition of UAV spraying droplets.

First of all, in the experiments under the same temperature and humidity environment conditions, experiments with numbers 1 and 2 (temperature $10^{\circ} \mathrm{C}$, humidity $60 \% \mathrm{RH}$ ) were respectively conducted on the first day morning (before cooling) and second day noon (after cooling). The two groups have different conditions such as light intensity and air pressure. According to the indications of the illuminance meter and the absolute pressure gauge in the wind tunnel, the meteorological conditions of the experiment of number 1 are: average illuminance of $20 \mathrm{Lux}$ and the average pressure of $108 \mathrm{kPa}$, and the meteorological conditions of experiment number 2 are: average illuminance of $150 \mathrm{Lux}$ and average pressure of $103 \mathrm{kPa}$. In the experiments with numbers 3 and 4 (temperature $19^{\circ} \mathrm{C}$, humidity $50 \% \mathrm{RH}$ ), the experiment with number 3 was carried out in the morning when the temperature was returned, and the experimental meteorological conditions were: average illuminance $10 \mathrm{Lux}$, average air pressure $108 \mathrm{kPa}$. The experiment with number 4 was conducted at noon after the temperature was lowered and its experimental meteorological conditions were: average illuminance 260 Lux and average pressure $105 \mathrm{kPa}$. The experimental result is that the difference between the two groups of experimental data is not significant. Therefore, it is proved that in the wind tunnel environment, in addition to temperature and humidity, other meteorological conditions have little effect on the deposition of droplets, which also provides an experimental basis for exploring the effect of temperature and humidity on droplets.

Secondly, in the experiment, errors will inevitably occur due to internal reasons for the machine and individual improper operation. Therefore, individual data may show errors that are inconsistent with the changing trend. Only through the analysis of many repeated experiments can we determine whether a certain data was wrong due to some factors. As for the different environment humidity experiment, although individual data may show a difference from the ideal value in the overall trend, and the fluctuation on the curve may be great in some places, the overall trend can be determined as we see from the data analysis. The experiment objects with experiment numbers 5-9 are the influence of humidity on the droplet deposition of UAV spraying operations. 
All environment temperatures in the experiment were $24^{\circ} \mathrm{C}$, and the humidities were $33 \% \mathrm{RH}, 45 \% \mathrm{RH}, 55 \% \mathrm{RH}, 68 \% \mathrm{RH}, 77 \% \mathrm{RH}$ respectively. The experimental results show that under the condition of $24^{\circ} \mathrm{C}$, with the increase of humidity, the droplet size, coverage, and deposition amount of the droplets sprayed by the UAV will increase. It shows that the environmental humidity has a great influence on the droplet deposition parameters.

Lastly, the experiments with serial numbers 10-12, 13-14, 15-16 are the effects of temperature. The three groups of experiments are: (1) The humidity is $54 \% \mathrm{RH}$, the temperatures are $13^{\circ} \mathrm{C}, 19^{\circ} \mathrm{C}, 29^{\circ} \mathrm{C}$. (2) Humidity is $68 \% \mathrm{RH}$, the temperatures are $11^{\circ} \mathrm{C}$ and $28^{\circ} \mathrm{C}$. (3) Humidity is $46 \% \mathrm{RH}$, the temperatures are $15^{\circ} \mathrm{C}$ and $24^{\circ} \mathrm{C}$. The result of the experiment is that the temperature condition has no significant effect on the droplet deposition of the UAV spraying. Because the experiment was conducted in winter, and the higher temperature could not be captured. The temperature range of the experiment is relatively small, the maximum temperature range is $10 \sim 29^{\circ} \mathrm{C}$. Therefore, the effect of higher temperatures on the deposition of droplets still needs to continue experimental research.

\section{Conclusion}

In this experiment, combined with the characteristics of the changing temperature in winter in Guangzhou, using the UAV experimental orbit platform, the effect of meteorological conditions on the droplet deposition of the UAV spraying operation was studied, and the following conclusions were drawn:

1) In wind tunnel conditions, except for temperature and humidity, the influence of air pressure and light intensity on the deposition of droplets are not significant.

2) Humidity conditions are likely to have a greater impact on the droplet size, coverage, deposition volume, and deposition density of droplets, as the droplet size, coverage, and deposition volume increase with increasing humidity. However, the deposition density tends to decrease due to polymerization when the droplet size is large. It is speculated that the reason is that under high humidity conditions, the air contains more water. On the one hand, it is easy to combine with the spray droplets in the air; On the other hand, the droplets in the air may drift towards the coated art paper under the action of the wind field of the UAV rotor. They may both cause the droplet deposition particle size, coverage, deposition amount and deposition density to increase.

3) In the temperature range of $10 \sim 29^{\circ} \mathrm{C}$ and the humidity range of $45-68 \% \mathrm{RH}$, the effect of temperature conditions on the droplet deposition of UAV spraying operations is not significant. The reason is speculated that the ambient temperature is much lower than the boiling point of the liquid, so it has little effect on the droplet size, coverage, deposition amount and deposition density of droplet.

4) The study still needed to be further because of the influence of the high outdoor temperature and a bigger range of the humidity have not been verified. Due to the lack of more experimental data, it is difficult to establish a model of temperature and humidity affecting UAV spray parameters. Sufficient experimental data is of great significance for building the spray model of unmanned agricultural aircraft system and improving the efficacy of UAV spraying.

\section{[References]}

[1] Xue X, Lan Y. Agricultural aviation applications in USA. Transactions of the Chinese Society of Agricultural Machinery, 2013, 44(5): 194-201. doi: 10.6041/j.issn.1000-1298.2013.05.034
[2] Lan Y B, Chen S D, Fritz B K. Current status and future trends of precision agricultural aviation technologies. International Journal of Agricultural and Biological Engineering, 2017, 10(3): 1-17. doi: 10.3965/j.ijabe.20171003.3088

[3] De Schampheleire M, Nuyttens D, Baetens K, et al. Effects on pesticide spray drift of the physicochemical properties of the spray liquid. Precision Agriculture, 2009, 10(5): 409-420. https://doi.org/10.1007/ s11119-008-9089-6

[4] Zhang J, Xie W, Zhou T, et al. Experimental Study on Spray Characteristics of Centrifugal Nozzle for Plant Protection UAV under Wind Tunnel Condition. China Southern Agricultural Machinery, 2019, 50(15): 2-8. (in Chinese)

[5] Tang Q, Chen L, Zhang R, et al. Droplet spectra and high-speed wind tunnel evaluation of air induction nozzles. Frontiers of Agricultural Science and Engineering, 2018, 5(4): 442-454.

[6] Lv M, Xiao S, Yu T, et al. Influence of UAV flight speed on droplet deposition characteristics with the application of infrared thermal imaging. International Journal of Agricultural and Biological Engineering, 2019, 12(3): 10-17. doi: 10.25165/j.ijabe.20191203.4868

[7] Ling W, Du C, Ze Y, et al. Research on the prediction model and its influencing factors of droplet deposition area in the wind tunnel environment based on UAV spraying. IFAC-PapersOnLine, 2018, 51(17) 274-279. https://doi.org/10.1016/j.ifacol.2018.08.174

[8] Ru L, Zhu C, Bao R. Spray Drift Model of Droplet and Analysis of Influencing Factors Based on Wind Tunnel. Transactions of the Chinese Society for Agricultural Machinery, 2014, 45(10): 66-72. doi: 10.6041/j.issn.1000-1298.2014.10.011 (in Chinese)

[9] Teske M E, Thistle H W, Riley C M, et al. Laboratory measurements of evaporation rate of droplets at low relative wind speed. Transactions of the ASABE, 2018, 61(3): 919-923. doi: 10.13031/trans.12743

[10] Wu J, Nofziger D L. Incorporating Temperature Effects on Pesticide Degradation into a Management Model. Journal of Environmental Quality, 1999, 28(1). https://doi.org/10.2134/ jeq1999.00472425002800010010x.

[11] Orlova-Bienkowskaja M J, Bieńkowski A O. Minimum Winter Temperature as a Limiting Factor of the Potential Spread of Agrilus planipennis, an Alien Pest of Ash Trees, in Europe. Insects, 2020, 11(4): 258. https://doi.org/10.3390/insects 11040258 .

[12] Alizadeh A, Bahadur V, Zhong S, et al. Temperature dependent droplet impact dynamics on flat and textured surfaces. Applied physics letters, 2012, 100(11): 111601. https://doi.org/10.1063/1.3692598

[13] Fedorets A A, Dombrovsky L A, Ryumin P I. Expanding the temperature range for generation of droplet clusters over the locally heated water surface. International Journal of Heat and Mass Transfer, 2017, 113: 1054-1058. https://doi.org/10.1016/j.ijheatmasstransfer.2017.06.015

[14] Binesh A R, Mousavi S M, Kamali R. Effect of temperature-dependency of Newtonian and non-Newtonian fluid properties on the dynamics of droplet impinging on hot surfaces. International Journal of Modern Physics C, 2015, 26(09): 1550106. https://doi.org/10.1142/ S0129183115501065

[15] Jin $\mathrm{Z}, \mathrm{Hu} \mathrm{H}$. Effect of surface temperature on droplet evaporation. Journal of Tongji University. Natural Science, 2012, 40(3): 495-498.

[16] Zuo Z, Wang J, Huo Y, et al. Numerical study of droplet evaporation in coupled high-temperature and electrostatic fields. Advances in Mechanical Engineering, 2015, 7(3). https://doi.org/10.1177/ 1687814015575388

[17] Portuguez E, Alzina A, Michaud P, et al. Evolution of a Water Pendant Droplet: Effect of Temperature and Relative Humidity. Natural Science, 2017, 09(1): 1-20. doi: 10.4236/ns.2017.91001

[18] Kubochkin N S, Ivanova N A. Droplet Shape and Wetting Behavior under the Influence of Cyclically Changing Humidity. Langmuir, 2019, 35(14): 5054-5059. doi: 10.1021/acs.langmuir.9b00159

[19] Jadidi M, Farzad M A, Trepanier J Y, et al. Effects of ambient air relative humidity and surface temperature on water droplet spreading dynamics. Fluids Engineering Division Summer Meeting. American Society of Mechanical Engineers, 2018, 51555: V001T15A006. doi: 10.1115/FEDSM2018-83287

[20] Bugarin L, Badhan A, Mao S. Numerical Investigation of Impact of Relative Humidity Condition on Droplet Accumulation and Film Cooling on Compressor Blades. Fluids Engineering Division Summer Meeting. American Society of Mechanical Engineers, 2014, 46216: V01AT02A008. https://doi.org/10.1115/FEDSM2014-21647

[21] Bou-Zeid W, Brutin D. Role of contact line evaporation on the spreading of viscous droplet. APS, 2014: M12. 009. 